\title{
Properties of Atmospheric Pressure Ar Plasma Jet Depending on Treated Dielectric Material
}

\author{
Vadym Prysiazhnyi $^{1}$ (D) A Alonso H. Ricci Castro ${ }^{1} \cdot$ Konstantin G. Kostov $^{1}$
}

Received: 24 August 2016 / Published online: 13 December 2016

(C) Sociedade Brasileira de Física 2016

\begin{abstract}
Atmospheric pressure plasma jet operated in argon was utilized to modify surfaces of glass, acrylic, and PTFE dielectrics. This paper describes the influence of the dielectric substrate on operation and properties of plasma. Two modes of operation (each of those have two patterns) were described. The transition from one mode to another, values of the dissipated power, and spreading of plasma over the dielectric surfaces strongly depended on the substrate material. Additionally, three methods of plasma spreading estimation were presented and discussed.
\end{abstract}

Keywords Ar plasma jet · Current patterns · Atmospheric pressure plasma jet $\cdot$ Electrical characterization $\cdot$ Plasma spreading

\section{Introduction}

The development of atmospheric pressure plasma jets (APPJs) has significantly increased in the last decades. Versatility of APPJs (configurations, sizes, gases or mixtures, powering) allowed finding various utilizations of these plasma sources. Surface cleaning and functionalization [1], plasma depositions [2], and applications of plasma in medicine [3] can be named as ones of many examples. Application of APPJs for modification of different substrates leads to a simple question,

Vadym Prysiazhnyi

vadym.p@protonmail.com

1 Department of Physics and Chemistry, Faculty of Engineering (campus Guaratinguetá), São Paulo State University (UNESP), Guaratinguetá, São Paulo, Brazil whether selected plasma jet will behave the same way while being in contact with substrates made of different materials. There were few reports describing different behavior of plasma jet depending on the substrate material. Hao et al. [4] showed differences in electrical characteristics of Ar plasma jet operated at same conditions (operation voltage, jet geometry, distance between the jet nozzle), but different dielectric substrate (glass, polytetrafluoroethylene (PTFE) or epoxy). Authors presented that the values of the dissipated power and current waveforms depended on material, though geometrical dimensions of samples (which, from our experience, play determining role for the actual values) were not mentioned. Detailed explanation of He plasma jet spreading over a glass substrate was presented by Guaitella et al. [5]. Correlating electrical measurements together with ICCD imaging, a propulsion of plasma was described. Norberg et al. [6] utilized modeling of plasma propagation and concentrations of several important species over dielectric and grounded metal surface. They show a significant effect of the substrate permittivity and its influence on the development of the discharge.

Surface wettability, on the other hand, is an important parameter that can indicate on the adhesion properties [7], biocompatibility [8], or ability to attach cells [9]. A comparative study of surface wettability of different materials after plasma treatments were presented by Toshifuji et al. [10], where the effect of pulsed gliding arc on the contact angle of metal and polymer materials was reported. They showed that wetting behavior after plasma application is strongly dependent on initial material; however, there were no detailed discussion of the presented results (variation of contact angle can be due to several changes: morphological, chemical, or both). Kim et al. [11] presented more material-focused study of APPJ treatment of several metals. Authors mention that the main effect on surface modification depends on oxygen species, and plasma induced changes on metal surfaces have short 
duration of about $15 \mathrm{~h}$ until aging affect takes place. The relationship between material properties and their influence on plasma was investigated in detail by Zaplotnik et al. [12]. The authors mentioned that, in the articles about surface modifications by plasma jets, this relationship is often omitted. They carefully described the changes in plasma impacted by the contact with different substrates: electrical properties of plasma and emission characteristics (including changes in quantity of active species, concentrations, and plasma parameters). Often, the substrate plays the role of an additional electrode and can be a source of vapors or sputtered material, and influence the chemistry inside plasma.

Other important issue that was not discussed directly is the spreading of plasma over a treated surface. Indirectly, this issue was highlighted when surface charging was studied. For example, Li et al. [13] demonstrated that PTFE surfaces kept higher charge density than alumina or quartz, which indirectly resulted in lower filament spreading that was confirmed below. Kostov et al. [14] studied an increase of surface wettability after an $\mathrm{Ar}$ plasma jet is extracted from a syringe. Authors showed that depending on the nozzle to surface distance, the area with improved wettability varies in a wide range. The study of treated area of PET surface was also reported by Onyshchenko et al. [15], showing that plasma jet modification extends over area that is much larger that the nozzle orifice. Especially, the plasma spreading is important when a jet with multiple nozzles is considered for uniform treatment of larger area [16]. Schlieren photography represents one more technique that can indirectly give certain understanding about the plasma spreading. Robert et al. [17] demonstrated that turning the discharge on introduces the turbulence, even when Reynolds number is in a range of a laminar flow. Boselli et al. [18] utilized this technique to study the influence of many operation parameters (including the presence of a substrate) on a gas dynamics. Metal substrates were characterized by propagation of a turbulent wave, while spreading of a dielectric substrate was mostly influenced by a surface charging.

We believe that the effect of substrate material on electrical properties of plasma and plasma spreading of APPJs were not highlighted and investigated with enough details. This article addresses those issues. The plasma spreading will be of a high interest for development of plasma jet arrays, when a homogeneous treatment of a large piece is a challenging task.

\section{Experimental Arrangements}

The APPJ treatments were done using a single electrode plasma jet with normally cut nozzle described in our previous work [19]. The experimental arrangement is presented in Fig. 1. It consists of a Pyrex tube (inner diameter of $3.3 \mathrm{~mm}$, outer diameter of $7.5 \mathrm{~mm}$ ), a tungsten rod high-voltage electrode, PTFE holder for the electrode, and gas inlet. The plasma is generated by $19 \mathrm{kHz}$ AC signal (Minipuls 4, Germany) with applied voltages varied from 10 to $18 \mathrm{kVp}$-p. Due to specific coupling between APPJ and power supply at $19 \mathrm{kHz}$, the signal is not a sine wave, but a combination of two sine-like signals (one with higher amplitude, second with lower). Ar (99.99\% purity) was used as a plasma gas. Three dielectric materials were treated using plasma. They are common glass (soda-lime-silica glass), PTFE plastic (cuts from a PTFE rod), and acrylic sheet. In order to make proper comparison, all samples made from the selected materials had $4 \mathrm{~mm}$ thickness. Glass and acrylic samples had a 4-cm $\times 4$-cm square shape, while the PTFE sample had a circular shape of $4 \mathrm{~cm}$ in diameter. Those samples were located on a $4 \mathrm{~mm}$ thick glass table with $50 \mathrm{~mm}$ aluminum circle attached from the bottom and used as a ground electrode. Electrical properties of the discharge were measured using Tektronix TDS 3032 oscilloscope. Voltage waveforms were obtained from a built-in exit from the generator (1:2000 divider). Current waveforms were recorded from a $47 \Omega$ inductance-free resistor connected in series. The dissipated power was obtained by the Lissajous figures by measuring the voltage on the $11 \mathrm{nF}$ capacitor connected in series. The low voltage from resistor and capacitor was acquired using Tektronix P2220 probe.

The spreading of plasma over the sample surface was evaluated using three approaches. First, the area of a $32 \mu \mathrm{l}$ water droplet over the treated surface was measured. Second, a distribution of water contact angle on the sample surface was studied by placing a $1 \mu \mathrm{l}$ volume of water drop each $4 \mathrm{~mm}$ from each other. To do this, a standard Ramé-Hart goniometer (model $100)$ was utilized. The system consists of a light source, a sample table with a syringe, and a camera. The water drop volume and the camera were controlled by computer using DropImage software. And, finally, third, the light intensity at the surface was analyzed on photos of the discharge using a conventional digital camera (Nikon D3100/Tamron 70-300) in partial (yet controlled) darkness.

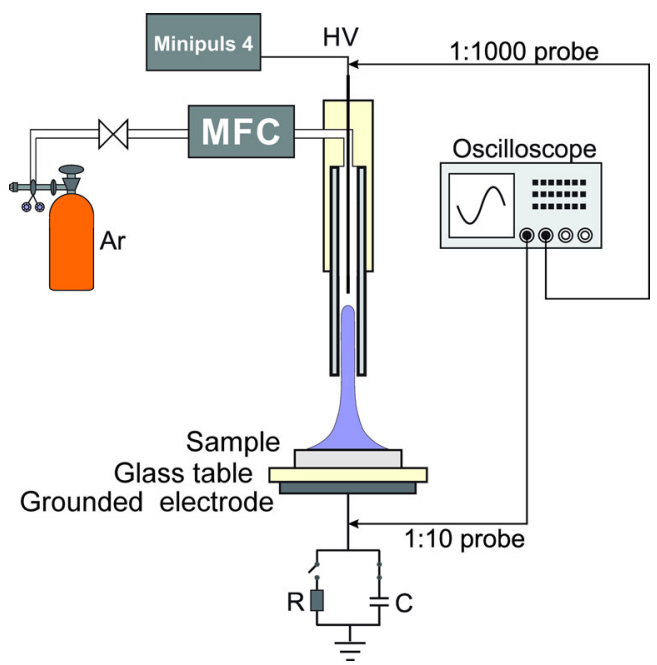

Fig. 1 A schema of the experimental setup 


\section{Results}

\subsection{Electrical Properties of APPJ Depending on the Substrate Material}

In order to analyze current waveforms, gas flow and nozzle-tosample distance were varied, while leaving the applied voltage at constant value of $15 \mathrm{kVp}$-p. The current waveforms for all three materials had related behavior. Similar current patterns were recently reported by Fang et al. [20], where honeycomblike jet array was characterized. It was found that similar to breakdown voltage of gas discharges, when a breakdown voltage is depending on combined parameter $p \cdot d$, the current changes is depending not only on the nozzle-to-sample distance or gas flow separately, but also on a combination of both. Figure 2 illustrates an example of current waveform evolution, when the glass substrate was in contact with plasma. For long distance or low gas flow, currents have only few distinct positive peaks of relatively high amplitude, and current at one negative peak appeared in some periods (Fig. 2a). With decrease of distance or increase of gas flow, the peaks appear in all periods, number of positive peaks increasing, and one distinct negative current peak appears (Fig. 2b). Further change of the mentioned above parameters led to transition of current pattern similar to filamentary dielectric barrier discharge (DBD) plasma [21] in positive voltage pulse only (Fig. 2c) and then in both positive and negative voltage pulse (Fig. $2 \mathrm{~d}$ ).

The transitions of current waveform mentioned above were observed for each studied material. However, it is rather complicated to determine the exact transition point, because for a certain region of gas flows and during a set of periods, two current patterns are presented. Table 1 represents the values of gas flow ranges, when a certain current pattern is dominant (pattern names are kept the same as assigned in Fig. 2) for a constant nozzle-to-substrate distance of $10 \mathrm{~mm}$. The results clearly show that current patterns transition strongly depends on the substrate material. Pattern " $D$ " (DBD-like) was detected for glass substrates and short nozzle-to-substrate distances only (less than $6 \mathrm{~mm}$ ).

We have observed that the jet power depends on the substrate material. As shown in Fig. 3, the values of power significantly depend on the material type. For example, the power of jet generated over the glass substrate can be over five times higher than the one over PTFE. For distances less than $3 \mathrm{~mm}$, the dissipated power saturates for all three materials, which is probably due to gas flow dynamics effects and surface charging. Limiting the distance between the nozzle and the substrate results in decrease of the plasma volume and faster dumping of a

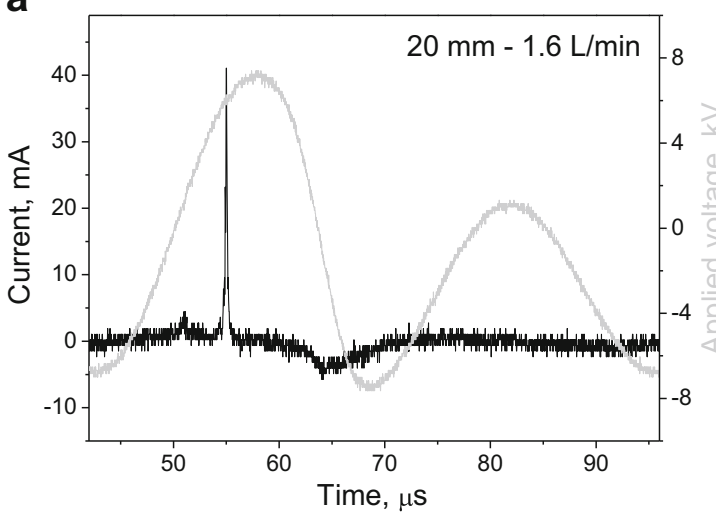

C

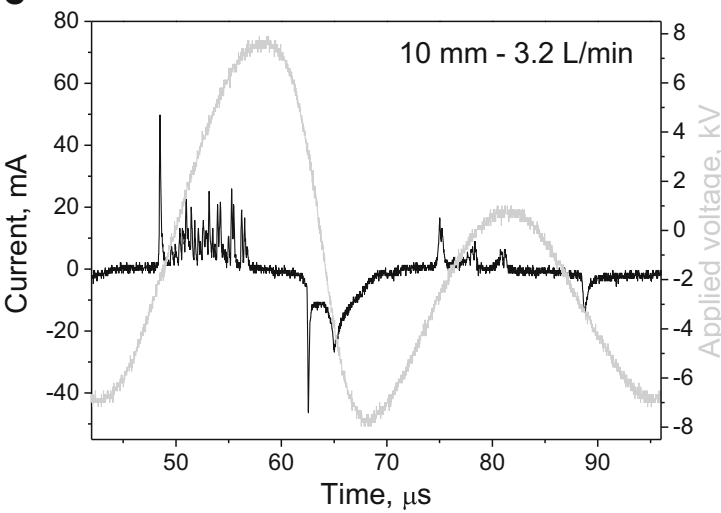

b

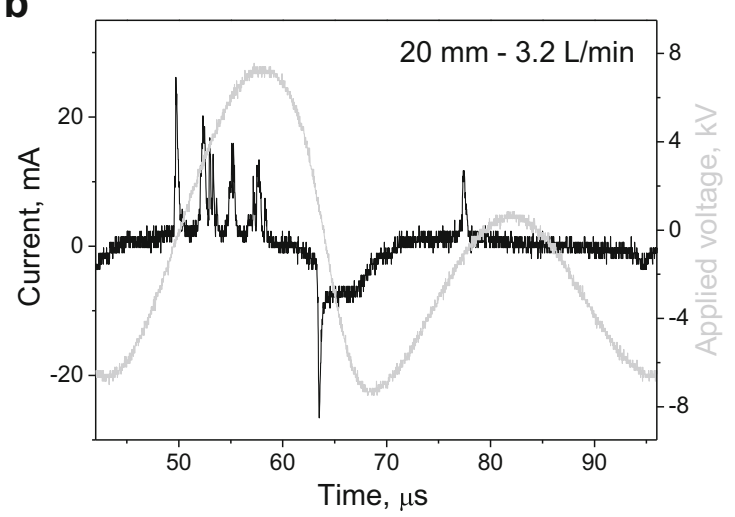

d

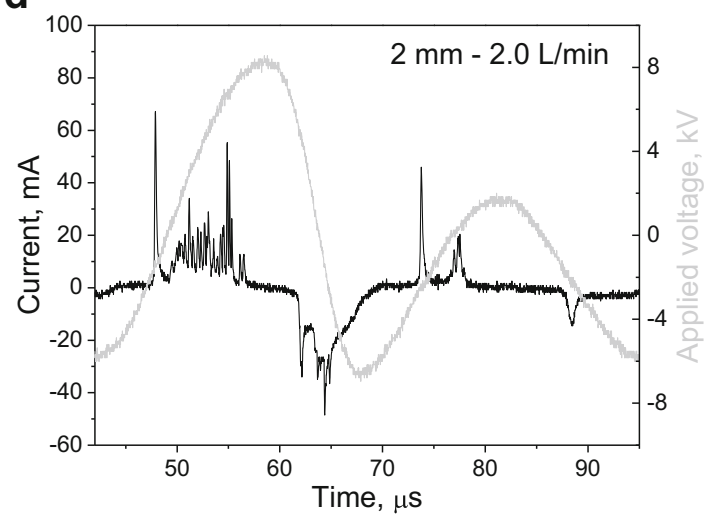

Fig. 2 Typical current behavior of Ar plasma jet in contact with glass substrate 
Table 1 Minimum gas flow values that correspond to a dominant pattern type (gas flow varied from 0.2 to $3.5 \mathrm{~L} / \mathrm{min}$ ) for nozzle-tosubstrate distance of $10 \mathrm{~mm}$

\begin{tabular}{llll}
\hline Substrate material & \multicolumn{2}{l}{$\begin{array}{l}\text { Starting gas flow (L/min), when a current } \\
\text { pattern is dominant }\end{array}$} \\
\cline { 2 - 4 } & Pattern "A" & Pattern "B" & Pattern "C" \\
\hline Glass & $0.2-1.0$ & $1.2-1.4$ & $1.6-3.5$ \\
Acrylic & $0.2-1.4$ & $1.6-3.5$ & - \\
PTFE & $0.2-1.6$ & $1.8-3.5$ & - \\
\hline
\end{tabular}

the plasma spreading which saturates the amount of transferred charge per voltage cycle. The plasma plume reached the substrate when nozzle-to-substrate distance was about $22 \mathrm{~mm}$, meaning that on this distance without light off, it is possible to observe that the end of the plume is located above the substrate. In this case, small changes in distance significantly affect the power values. When the plasma jet is located far away from the substrate, i.e., the plasma plume does not touch the substrate, air gap represents as an additional capacitor. Therefore, increasing the distance will lead to only gradual decrease of the calculated power, while raising a point whether $\mathrm{Q}-\mathrm{V}$ plot method is valid for this purpose on a large nozzle to substrate distances.

\subsection{Plasma Spreading}

Placing a large volume of liquid (tenth of $\mu \mathrm{l}$, comparing with few $\mu \mathrm{l}$ for a sessile-drop method) on the sample surface leads to spreading over big area. We have found that droplet of $32 \mu \mathrm{l}$ is large enough to reveal the area with enhanced wettability, while being not too heavy to affect the wetted area by gravity. The diameter of the area with enhanced wettability depended on the plasma treatment conditions. Table 2 presents the results for $5 \mathrm{~s}$ plasma treatment for all substrate types.

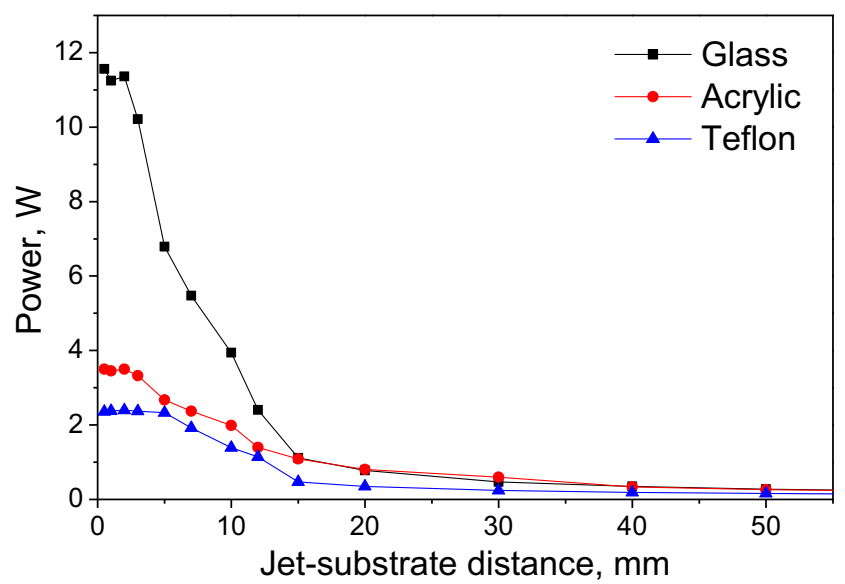

Fig. 3 The dissipated power as a function of the distance between the jet nozzle and the substrate and substrate material; gas flow, $0.8 \mathrm{~L} / \mathrm{min}$; applied voltage, $15 \mathrm{kVp}-\mathrm{p}$
Table 2 Diameter of $32 \mu$ water droplet over a sample surface treated for $5 \mathrm{~s}$ at $10 \mathrm{~mm}$ distance

\begin{tabular}{llrl}
\hline \multirow{2}{*}{ Gas flow, L/min } & \multicolumn{3}{l}{ Water droplet spreading, mm } \\
\cline { 2 - 4 } & \multicolumn{1}{l}{ PTFE } & \multicolumn{1}{c}{ Acrylic } & \multicolumn{1}{c}{ Glass } \\
\hline Untreated & $5.0 \pm 0.2$ & $7.0 \pm 0.2$ & $7.5 \pm 0.2$ \\
0.4 & $6.2 \pm 0.3$ & $7.5 \pm 0.5$ & $17 \pm 0.4$ \\
0.8 & $6.6 \pm 0.5$ & $8.0 \pm 0.5$ & $22 \pm 0.6$ \\
1.6 & $7.0 \pm 0.4$ & $10.5 \pm 0.6$ & $27 \pm 1.5$ \\
\hline
\end{tabular}

Using a small water droplet volume allows performing study of the contact angle value distribution along the sample. Three values of Argas flow for a constant distance between the nozzle and the sample were used. A typical water contact angle profile of the plasma treated acrylic samples is shown in Fig. 4.

Overall results of the measurements are presented in Table 3. The value of the WCA only slightly decreases with increasing the Ar gas flow, meaning that the treatment is close to saturation in the center area located just about the jet nozzle.

The light emission is the other characteristic that can be used to estimate the area treated by plasma jets. For APPJs, with respect to the plasma plume shape, the spread of plasma over the dielectric substrate has approximately circular form. The spread is as reported in the work of Wang et al. [22], where a circular etching of parylene film of Si substrate was recorded. The diameter of spreading can be determined using brightness analysis. In this work, we utilize ImageJ software and get the brightness profile from the surface; photos were taken perpendicularly (see Fig. 5a). The time of exposition for all photos was fixed to $40 \mathrm{~ms}$, and the border for the emission was set to $10 \%$ of the maximum intensity (see Fig. $5 b$ ).

We were particularly interested to find whether the material type would significantly influence the light emission spreading. The experiment was performed for three sample configurations: (i) only dielectric substrate (sample was placed on

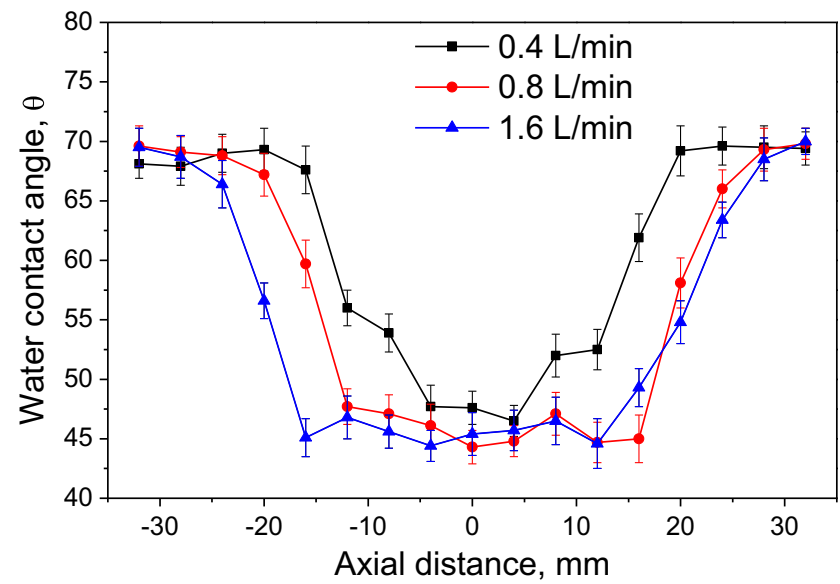

Fig. 4 Profile of water contact angle. Measurements were done for acrylic substrate, $10 \mathrm{~mm}$ nozzle-to-substrate distance and $15 \mathrm{kVp}-\mathrm{p}$ applied voltage 
Table 3 Area of improved wettability estimated by sessile-drop technique (treatment duration for $5 \mathrm{~s}$ at $10 \mathrm{~mm}$ nozzle-to-sample distance)

\begin{tabular}{clcl}
\hline & $\begin{array}{l}\text { Ar flow, } \\
\text { L/min }\end{array}$ & $\begin{array}{l}\text { WCA after } \\
\text { treatment, }\end{array}$ & $\begin{array}{l}\text { Diameter of area } \\
\text { with enhanced } \\
\text { wettability, mm }\end{array}$ \\
\hline Glass (untreated & 0.4 & 8 & 18 \\
WCA 38 ${ }^{\circ}$ & 0.8 & 6 & 32 \\
Acrylic (untreated & 0.4 & 5 & 44 \\
WCA 70 $)$ & 0.8 & 45 & 18 \\
& 1.6 & 45 & 28 \\
PTFE (untreated & 0.4 & 102 & 34 \\
WCA $123^{\circ}$ ) & 0.8 & 98 & 10 \\
& 1.6 & 83 & 14 \\
\hline
\end{tabular}

$4 \mathrm{~mm}$ thick glass table), (ii) floating metal electrode placed under the glass table, and (iii) grounded metal electrode placed below the glass table. The experimental values for each applied voltage/material were calculated as average of five photos. Typical diameter of bright area for PTFE substrate is presented in Fig. 6 as an example. As expected at the same voltages, the extraction of the plume was significantly facilitated when the discharge was ignited over the grounded sample table. In addition, using grounded table resulted in plasma ignition at lower applied voltages (from $10 \mathrm{kVp}-\mathrm{p}$ ). Adding a floating metal electrode only slightly influenced the plasma spreading over acrylic, while for the glass and the PTFE samples, it was insignificant. Table 4 presents the light emission diameter for three values of applied voltage.

\section{Discussion}

The electrical behavior (i.e., current patterns and value of the dissipated power of plasma jets) strongly depends on the substrate material. The studied single electrode Ar APPJ tends to work in two distinct modes that depend on a parameter "nozzle-to-sample distance/gas flow." This parameter represents the coupling between the plasma plume and the substrate. When coupling is low (nozzle-to-sample distance is high or gas flow is low), the current tends to have relatively short, high amplitude peaks with duration varied from $500 \mathrm{~ns}$ to $1 \mu \mathrm{s}$. When coupling is high, the current pattern is similar to the one observed in dielectric barrier discharges. In that case, a large number of short current peaks were observed (from about 100 to $200 \mathrm{~ns}$ ). The transition from one to other pattern depends on the material type. For all materials, the transition occurs first for positive current peaks and then for the negative ones.

The value of the dissipated power is a key control parameter for material treatment (in both material processing and especially for bio-medical applications) [23, 24]. It is important to note that changing the material can cause significant variation in the amounts of the discharge power. In our case, changing glass substrate to PTFE resulted in power decrease of five times compared to glass.

Estimation of the area affected by plasma jets is an important issue that is often omitted. Several research groups invested efforts to study and develop 1D (linear [25]) or 2D (honeycomb [26]) jet arrays. There are several issues when several plasma jets are located close to each other: changes in electric field (electric coupling) and new gas flow dynamics. It results in jet interactions that often lead to non-uniform jet array operation. Surface wettability represents a strong indicator for many technological applications like adhesion of coatings and painting and characterizes surface bio-compatibility. The simplest approach to check the wetting of larger surface is to use a water spray and observe whether small drops or continuous film is formed. However, this approach lacks reproducibility and can be utilized mostly as a demonstration experiment. We tested three approaches on how to estimate the plasma treated area. Two of them were related to the changes of the surface wettability. The sessile-drop technique (or contact angle measurement) is probably one of
Fig. 5 Plasma spreading determination using a brightness profile: a perpendicularly taken photo of plasma jet and $\mathbf{b}$ brightness profile obtained by ImageJ. Substrate material, glass; sample table, grounded; nozzlesubstrate distance, $10 \mathrm{~mm}$; Ar gas flow, $0.8 \mathrm{~L} / \mathrm{min}$; applied voltage, $14 \mathrm{kVp}-\mathrm{p}$
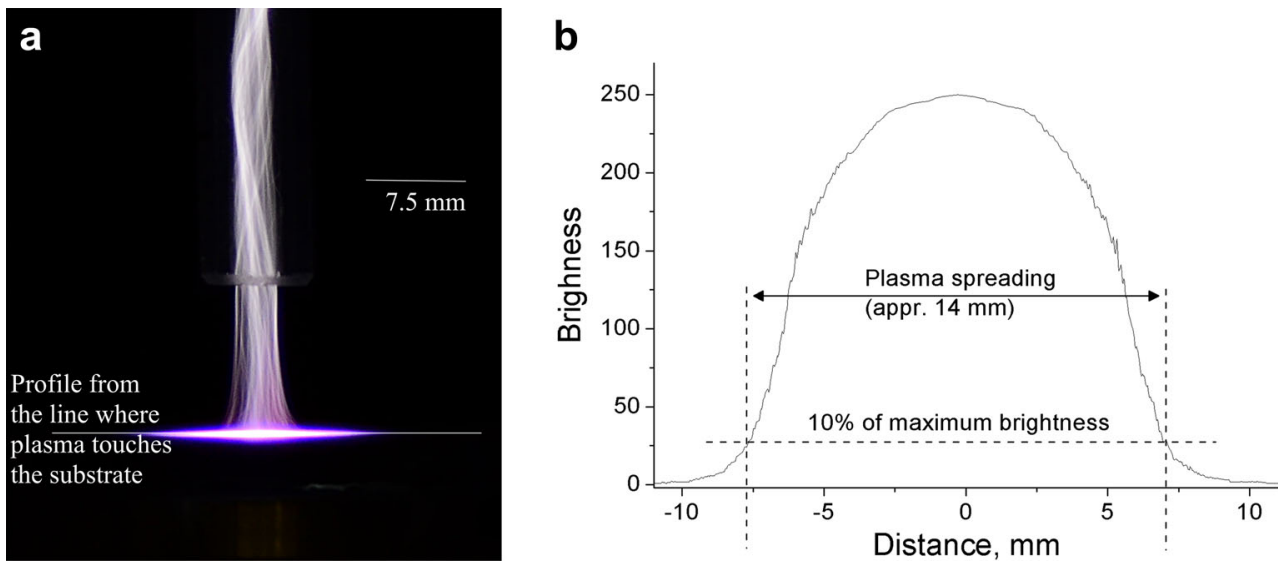


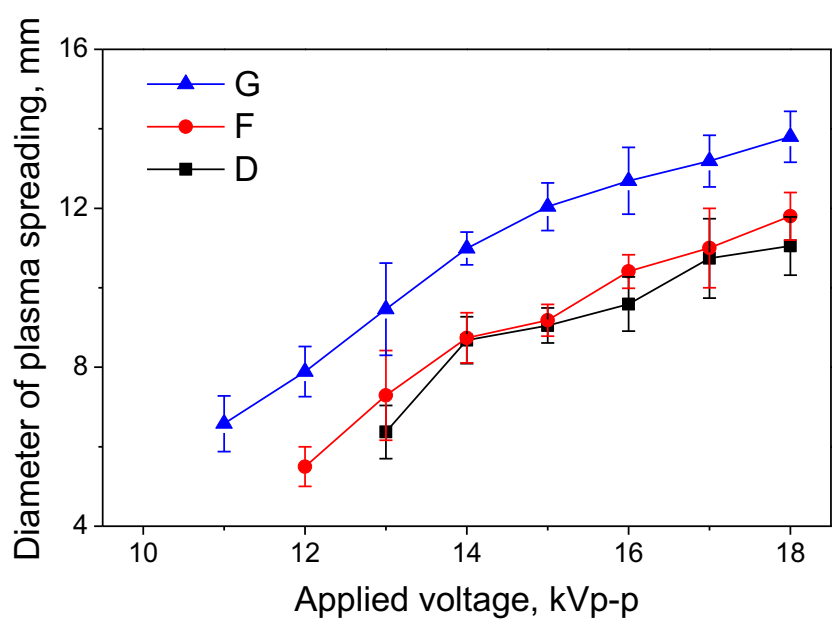

Fig. 6 Light emission spreading for PTFE. $D$ dielectric only, $F$ floating metal electrode, $G$ grounded metal electrode. Ar gas flow, $0.8 \mathrm{~L} / \mathrm{min}$; nozzle-to-substrate distance, $10 \mathrm{~mm}$

the most used methods to determine the surface wettability. And another way is placing a large water drop on the surface. We also utilize the brightness emission analysis to correlate the obtained results. Utilizing the scanning contact angle measurements, it was shown that three materials were treated differently under same condition. The treatment led to a decrease of WCA, though significant differences were observed in the values of the WCA. Glass surface became almost super-hydrophilic, acrylic polymer showed a reasonable decrease in the contact angle value, while PTFE almost did not changed its wettability. Other important observation is the growth of treated area. Increasing the flow four times led to almost six times higher treatment area (more than twice increase of the radius with enhanced wettability) for glass. For acrylic, the increase of the area was about 3.5, while the PTFE surface showed an increase a bit less than 2 times.

Comparing different methods of plasma spreading for each studied material was different. Table 5 shows combined values for same treatment condition estimated by three methods. The values obtained by the sessile-drop technique

Table 4 Light emission values

Water droplet spreading, $\mathrm{mm}$

\begin{tabular}{|c|c|c|c|c|c|c|c|c|}
\hline \multicolumn{3}{|c|}{ Glass sample } & \multicolumn{3}{|c|}{ Acrylic sample } & \multicolumn{3}{|c|}{ PTFE sample } \\
\hline $\mathrm{D}$ & $\mathrm{F}$ & G & $\mathrm{D}$ & $\mathrm{F}$ & $\mathrm{G}$ & $\mathrm{D}$ & $\mathrm{F}$ & G \\
\hline 10 & 12 & 14.9 & 7 & 9.2 & 11.3 & 6.4 & 7.3 & 9.5 \\
\hline 15 & 15.5 & 17.9 & 7.8 & 10.2 & 14.4 & 9 & 9.2 & 12.5 \\
\hline 17.8 & 17.9 & 21.1 & 9.1 & 11.3 & 18.5 & 10.7 & 11 & 13.1 \\
\hline
\end{tabular}

Ar gas flow, $0.8 \mathrm{~L} / \mathrm{min}$; nozzle-to-substrate distance, $10 \mathrm{~mm}$; D, sample table without metal; F, sample table with floating metal electrode; G, sample table with grounded metal electrode. Error of measurements was less than $2 \mathrm{~mm}$
Table 5 Comparative values of enhanced wettability area for $15 \mathrm{kV}$ applied voltage and $0.8 \mathrm{~L} / \mathrm{min}$ Ar gas flow

\begin{tabular}{llll}
\hline & $\begin{array}{l}\text { Sessile-drop } \\
\text { scanning }\end{array}$ & $\begin{array}{l}\text { Light intensity } \\
\text { spreading }\end{array}$ & $\begin{array}{l}\text { Large volume } \\
\text { drop spreading }\end{array}$ \\
\hline Glass & 32 [highest] & 18 [lowest] & 22 \\
Acrylic & 28 [highest] & 14.4 & 8 [lowest] \\
PTFE & 12 & 12.5 [highest] & 6.6 [lowest] \\
\hline
\end{tabular}

showed the largest affected by plasma area for most treatment conditions. However, note that each material demonstrated different values estimated by different approaches. It seems that surface wettability and its estimation cannot be simplified, as wettability area estimated using contact angle measurement scanning was always higher than emission of light over the same material.

\section{Conclusion}

Single electrode Ar atmospheric pressure plasma jet has two distinct operation modes while being in contact with a dielectric substrate: low- and high-gas coupling. Due to different behavior of current filaments in positive and negative voltages, each operation mode has two current patterns. Therefore, four current patterns can be expected for similar jets operated in Ar also. The change of pattern depends on three major parameters: substrate material, plasma jet to substrate distance, and gas flow. The first one determines the interaction between charged particles generated in plasma and surface charging. The other two parameters determine a coupling between the substrate and the plasma plume.

The substrate material strongly influences the value of the dissipated power in the plasma jet. This is an important finding, as in the majority of reports; the parameter that is used to quantitatively estimate the degree of modification is plasma treatment time, though, if different materials are treated, it is necessary to substitute the treatment time by the amount of deposited energy (power multiplied by time) in order to compare the APPJ efficiency for different materials. As an example, it has been shown that changing from glass to PTFE substrate keeping same operation parameters led to a fivetime reduction in dissipated power.

Surface wettability is an important surface characteristic and its value directly influences many processes, like adhesion of paintings and coatings, and several bio-applications. It is known that plasma treatments modify surface wettability (by cleaning, roughening, functionalization or a combination of those), and APPJ systems, due to their construction, alter only some limited surface areas. Ar plasma jets in ambient air typically increase the surface wettability. Three methods to estimate the area with enhanced wettability, scanning of contact 
angle, light emission measurement, or area of a large-volume drop were employed. It has been shown that material significantly influences the obtained areas after certain plasma treatment conditions. Though the highest area of the enhanced wettability was obtained for scanning the contact angle over the surface, each material showed different comparative values of each used approach. The obtained data of area modified by APPJ area were not presented to claim or state, rather to put a question, what is the most appropriate approach to determine the surface wettability? As for now, the obtained data showed that the nature of treated material strongly influences the plasma modification, difficulting an easy answer.

Acknowledgements This research was supported by Fundação de Amparo à Pesquisa do Estado de São Paulo (FAPESP: 2013/06732-3 and 2015/21989-6). Authors are grateful to Jose B. Galhardo for technical support.

\section{References}

1. Y. Jin, C.-S. Ren, L. Yang, D. Wang, IEEE T. Plasma Sci. 43, 3193 (2015)

2. X. Deng, A.Y. Nikiforov, N. De Geyter, R. Morent, C. Leys, Plasma Proces. Polym. 10, 641 (2013)

3. M. Laroussi, IEEE T. Plasma Sci. 43, 703 (2015)

4. Z. Hao, S. Ji, A. Qiu, IEEE T. Plasma Sci. 40, 2822 (2012)

5. O. Guaitella, A. Sobota, J. Phys. D. Appl. Phys. 48, 255202 (2015)

6. S.A. Norberg, E. Johnsen, M.J. Kushner, J. Appl. Phys. 118, $013301(2015)$

7. I. Novák, J. Sedliačik, M. Gajtanska, J. Schmidtová, A. Popelka, P. Bekhta, T. Krystofiak, S. Proszyk, O. Žigo, Bioresources 11, 6335 (2016)
8. J.L. Lauer, J.L. Shohet, R.M. Albrecht, C. Pratoomtong, R. Murugesan, S. Esnault, J.S. Malter, U.H. von Andrian, R.D. Bathke, S.B. Shohet, J. Appl. Phys. 96, 4539 (2004)

9. L.-C. Xua, C.A. Siedlecki, Biomaterials 28, 3273 (2007)

10. J. Toshifuji, T. Katsumata, H. Takikawa, T. Sakakibara, I. Shimizu, Surf. Coat. Technol. 171, 302 (2003)

11. M.C. Kim, S.H. Yang, J.-H. Boo, J.G. Han, Surf. Coat. Technol. 174-175, 839 (2003)

12. R. Zaplotnik, M. Bišćan, Z. Kregar, U. Cvelbar, M. Mozetič, S. Milošević, Spectrochim. Acta B 103-104, 124 (2015)

13. M. Li, C. Li, H. Zhan, J. Xu, X. Wang, Appl. Phys. Lett. 92, 031503 (2008)

14. K.G. Kostov, T.M.C. Nishime, A.H.R. Castro, A. Toth, L.R.O. Hein, Appl. Surf. Sci. 314, 367 (2014)

15. I. Onyshchenko, A.Y. Nikiforov, N. De Geyter, R. Morent, Plasma Process. Polym. 12, 466 (2015)

16. Q.Y. Nie, Z. Cao, C.S. Ren, D.Z. Wang, M.G. Kong, New J. Phys. 11, 115015 (2009)

17. E. Robert, V. Sarron, T. Darny, D. Ries, S. Dozias, J. Fontane, L. Joly, J.-M. Pouvesle, Plasma Sources Sci. Technol. 23, 012003 (2014)

18. M. Boselli, V. Colombo, E. Ghedini, M. Gherardi, R. Laurita, A. Liguori, P. Sanibondi, A. Stancampiano, Plasma Chem. Plasma Process. 34, 853 (2014)

19. A.H.R. Castro, K.G. Kostov, V. Prysiazhnyi, IEEE T. Plasma Sci. 43, $3228(2015)$

20. Z. Fang, C. Ruan, T. Shao, C. Zhang, Plasm. Sour. Sci. Technol. 25, 01LT01 (2016)

21. F. Massines, G. Gouda, J. Phys. D. Appl. Phys. 31, 3411 (1998)

22. T. Wang, B. Yang, X. Chen, X. Wang, C. Yang, J. Liu, Plasma Process. Polym. (2016). doi:10.1002/ppap.201600067

23. E.A. Sosnin, E. Stoffels, M.V. Erofeev, I.E. Kieft, S.E. Kunts, IEEE T. Plasma Sci. 32, 1544 (2004)

24. C.Z. Liu, J.Q. Wu, L.Q. Ren, J. Tong, J.Q. Li, N. Cui, N.M.D. Brown, B.J. Meenan, Mater. Chem. Phys. 85, 340 (2004)

25. Z. Cao, J.L. Walsh, M.G. Kong, Appl. Phys. Lett. 94, 021501 (2009)

26. J.Y. Kim, J. Ballato, S.-O. Kim, Plasma Process. Polym. 9, 253 (2012) 